\title{
Dielectric function of the QCD vacuum
}

\author{
H. Reinhardt* \\ Institut für Theoretische Physik \\ Auf der Morgenstelle 14 \\ D-72076 Tübingen \\ Germany \\ (Dated: October 31, 2018)
}

\begin{abstract}
It is shown that the inverse of the ghost form factor in the Hamilton approach to Yang-Mills theory in Coulomb gauge can be interpreted as the color dielectric function of the QCD vacuum. Furthermore the horizon condition to the ghost form factor implies that in the infrared the QCD vacuum is a perfect color diaelectric medium and therefore a dual superconductor. The dielectric function is explicitly calculated within a previously developed variational approach, using a specific ansatz for the vacuum wave functional.
\end{abstract}

PACS numbers: $11.10 \mathrm{Ef}, 12.38 \mathrm{Aw}, 12.38 \mathrm{Cy}, 12.38 \mathrm{Lg}$

\section{INTRODUCTION}

Soon after the discovery of QCD it was realized that due to the confinement of color charges the $\mathrm{QCD}$ vacuum must behave like a perfect (or nearly perfect) color diaelectric medium [1]. This picture is also realized in the MIT bag model [2] or SLAC bag model 3]. However, so far, no real attempt has been undertaken to calculate the color dielectric function of the QCD vacuum from the underlying theory. Obviously, such calculations of vacuum properties have to be nonperturbative.

The QCD vacuum and, in particular, the confinement mechanism have been subject to intensive studies, from which several pictures of confinement have emerged like the dual Meissner effect [4], center vortex condensation [5], 6] or the Gribov-Zwanziger confinement mechanism [], [8]. Although there is evidence from lattice calculations that the various confinement pictures - in particular the first two - are related no direct connection between these pictures have been established so far. In the present paper I show that the Gribov-Zwanziger confinement scenario in Coulomb gauge implies the dual superconductor. This connection is based on the observation made in sect. III that the inverse of the ghost from factor in Coulomb gauge represents the dielectric function of the Yang-Mills vacuum.

Recently, progress has been made in determining the Yang-Mills vacuum wave functional by a variational solution of the Yang-Mills Schrödinger equation inCoulomb gauge [9], [10]. The infrared properties calculated with the wave functional obtained in refs. 10], 11] and [12] show clear signals of confinement: an infrared divergent gluon energy, a linearly rising static quark potential and a perimeter law for the 't Hooft loop [13]. Therefore, we may expect that the variationally determined vacuum wave functional contains the essential infrared physics of the Yang-Mills vacuum. In the present paper I shall use

*Electronic address: hugo.reinhardt@uni-tuebingen.de this wave functional to explicitly calculate the dielectric function of the QCD vacuum. I will first identify the dielectric function in the Hamilton approach in Coulomb gauge and discuss some of its general properties, which do not rely on the variational approach. After that I will present the numerical results for this quantity obtained by using the wave functional determined in ref. [11].

\section{THE HAMILTON APPROACH TO YANG-MILLS THEORY IN COULOMB GAUGE}

Consider the Hamilton formulation of Yang-Mills theory in Weyl gauge $A_{0}^{a}(\boldsymbol{x})=0$. The electric field $E_{i}^{a}$ represents the momentum conjugate to the spatial components of the gauge field $A_{i}^{a}$. In the canonical quantization the electric field is replaced by the momentum operator $\Pi_{i}^{a}(\boldsymbol{x})=\frac{1}{i} \frac{\delta}{\delta A_{i}^{a}(\boldsymbol{x})}$ and the Yang-Mills Hamiltonian reads

$$
H=\frac{1}{2} \int d^{3} x\left(\boldsymbol{\Pi}^{2}(\boldsymbol{x})+\boldsymbol{B}^{2}(\boldsymbol{x})\right),
$$

where $B_{i}^{a}$ is a non-abelian magnetic field.

In Weyl gauge, Gauss' law is lost from the equation of motion and has to be imposed as a constraint to the wave functional

$$
\hat{D}_{i}^{a b} \Pi_{i}^{b}(\boldsymbol{x})|\psi\rangle=g \rho^{a}(\boldsymbol{x})|\psi\rangle .
$$

Here $\hat{D}_{i}^{a b}=\delta^{a b} \partial_{i}+g \hat{A}_{i}^{a b}, \hat{A}^{a b}=f^{a c b} A^{c}$ is the covariant derivative in the adjoint representation of the gauge group ( $f^{a b c}$ being the structure constant of the gauge group and $g$ being the coupling constant) and $\rho^{a}(\boldsymbol{x})$ denotes the "external" color charge density of the matter fields. The operator on the left hand side of eq. (2) is the generator of time-independent gauge transformations and thus Gauss' law expresses the gauge invariance of the wave functional.

Instead of working with gauge invariant wave functionals it is more convenient to explicitly resolve Gauss' law by fixing the gauge. For this purpose, Coulomb gauge $\boldsymbol{\partial} \boldsymbol{A}^{a}=0$ is a particularly convenient gauge, which will 
be used in the following. In Coulomb gauge only the transversal components of the gauge field $A=A_{\perp}$ are left. Splitting the momentum operator in longitudinal and transversal parts $\Pi=\Pi_{\|}+\Pi_{\perp}, \Pi_{\perp}=\frac{1}{i} \delta / \delta A_{\perp}^{a}$, respectively, Gauss' law can be solved for the longitudinal part yielding

$$
\boldsymbol{\Pi}_{||}|\psi\rangle=-g \boldsymbol{\partial}(-\hat{\mathbf{D}} \boldsymbol{\partial})^{-1}\left(\rho+\rho_{g}\right)|\psi\rangle,
$$

where $\rho_{g}^{a}=-\hat{A}_{\perp i}^{a b} \Pi_{\perp i}^{b}$ is the color charge density of the gauge bosons and ( $-\hat{\mathbf{D}} \boldsymbol{\partial})$ is the Faddeev-Popov kernel in Coulomb gauge.

We are interested in the electric field which in the quantum theory is defined as the expectation value of the corresponding (momentum) operator

$$
\boldsymbol{E}=\langle\boldsymbol{\Pi}\rangle=\left\langle\boldsymbol{\Pi}_{\|}+\boldsymbol{\Pi}_{\perp}\right\rangle .
$$

The vacuum wave functional can be assumed to be invariant with respect to the transformation $A_{\perp} \rightarrow-A_{\perp}$, implying that $\left\langle\boldsymbol{\Pi}_{\perp}\right\rangle=0$. With this result we obtain from eq. (3) for the electric field

$$
\boldsymbol{E}=\left\langle\boldsymbol{\Pi}_{\|}\right\rangle=-\boldsymbol{\partial}\left(g\left\langle(-\hat{\mathbf{D}} \boldsymbol{\partial})^{-1}\right\rangle \rho+g\left\langle(-\hat{\mathbf{D}} \boldsymbol{\partial})^{-1} \rho_{g}\right\rangle\right) .
$$

Here the first term represents the response of the YangMills vacuum to the presence of the external color charges $\rho^{a}(\boldsymbol{x})$. In the absence of external color charges $\rho^{a}(\boldsymbol{x})=0$ the Yang-Mills vacuum should not contain any observable color electric field, i.e. $\langle\Pi\rangle_{\rho=0}=0$, from which we can conclude that the second term in eq. (5) vanishes in the Yang-Mills vacuum state. The color electric field generated by external color charges $\rho^{a}(\boldsymbol{x})$ is therefore given by

$$
\boldsymbol{E}^{a}(\boldsymbol{x})=-\boldsymbol{\partial}_{\boldsymbol{x}} \int d^{3} x^{\prime} G^{a b}\left(\boldsymbol{x}, \boldsymbol{x}^{\prime}\right) \rho^{b}\left(\boldsymbol{x}^{\prime}\right),
$$

where

$$
G^{a b}\left(\boldsymbol{x}, \boldsymbol{x}^{\prime}\right)=\left\langle(-\hat{\mathbf{D}} \boldsymbol{\partial})^{-1}\right\rangle\left(\boldsymbol{x}, \boldsymbol{x}^{\prime}\right)
$$

is the ghost propagator. Let us emphasize that eq. (6) has exactly the same structure as the electric field generated by an ordinary electric charge density $\rho(\boldsymbol{x})$ in classical electrodynamics

$$
\begin{aligned}
\boldsymbol{E}(\boldsymbol{x}) & =-\boldsymbol{\partial}_{\boldsymbol{x}} \int d^{3} x^{\prime}\left\langle\boldsymbol{x}\left|(-\Delta)^{-1}\right| \boldsymbol{x}^{\prime}\right\rangle \rho\left(\boldsymbol{x}^{\prime}\right) \\
& =-\boldsymbol{\partial}_{\boldsymbol{x}} \int d^{3} x^{\prime} \frac{\rho\left(\boldsymbol{x}^{\prime}\right)}{4 \pi\left|\boldsymbol{x}-\boldsymbol{x}^{\prime}\right|}
\end{aligned}
$$

except that the Green function of the Laplacian is replaced by the ghost propagator. 23.

\section{THE DIELECTRIC FUNCTION}

The ghost propagator, eq. (7), is a property of the Yang-Mills vacuum (and does not depend on the external charges). By global color invariance of the Yang-Mills vacuum this propagator has to be color-diagonal. Furthermore, by translational invariance of the Yang-Mills vacuum $G^{a b}\left(\boldsymbol{x}, \boldsymbol{x}^{\prime}\right)$ depends only on $\left|\boldsymbol{x}-\boldsymbol{x}^{\prime}\right|$. In momentum space the ghost propagator has therefore the following form

$$
g G^{a b}(\boldsymbol{k})=\delta^{a b} \frac{d(\boldsymbol{k})}{\boldsymbol{k}^{2}} .
$$

Here we have introduced the ghost form factor $d(\boldsymbol{k})$, which describes the deviations of the ghost propagator from the Green function of the Laplacian, i.e. it embodies all the deviations of the Yang-Mills vacuum from the QED case. With (9) we find from eq. (6) for the electric field in momentum space

$$
\boldsymbol{E}^{a}(\boldsymbol{k})=-i \boldsymbol{k} \frac{d(\boldsymbol{k})}{\boldsymbol{k}^{2}} \rho^{a}(\boldsymbol{k}),
$$

where $\boldsymbol{E}^{a}(\boldsymbol{k})$ and $\rho^{a}(\boldsymbol{k})$ denote the Fourier transform of $\boldsymbol{E}^{a}(\boldsymbol{x})$ and $\rho^{a}(\boldsymbol{x})$, respectively. Equation (10) should be compared with the expression for the ordinary electric field in a medium

$$
\boldsymbol{E}(\boldsymbol{k})=-i \boldsymbol{k} \frac{1}{\epsilon(\boldsymbol{k}) \boldsymbol{k}^{2}} \rho(\boldsymbol{k}),
$$

where $\epsilon(\boldsymbol{k})$ is the (generalized) dielectric function [14] and $\rho(\boldsymbol{k})$ the Fourier transform of the electric charge density. Comparing eqs. (10) and (11) we can identify the inverse of the ghost form factor as the dielectric function of the Yang-Mills vacuum

$$
\epsilon(\boldsymbol{k})=d^{-1}(\boldsymbol{k}) .
$$

In QED the Faddeev-Popov kernel of Coulomb gauge is given by the Laplacian and the ghost form factor is $d(\boldsymbol{k})=1$, so that $\epsilon(\boldsymbol{k})=1$ in the QED vacuum, as expected.

On general grounds one expects that the ghost form factor in QCD is infrared divergent

$$
d^{-1}(\boldsymbol{k}=\mathbf{0})=0 .
$$

This is the so-called horizon condition, which is required for confinement in the Gribov-Zwanziger confinement scenario 7], 8]. By this condition the dielectric function of the Yang-Mills vacuum (12) vanishes in the infrared

$$
\epsilon(\boldsymbol{k}=\mathbf{0})=0
$$

and hence the Yang-Mills vacuum is a perfect color diaelectric medium.

A perfect color diaelectric medium $\epsilon=0$ is nothing but a dual superconductor (where dual refers to an interchange of electric and magnetic fields and charges). Recall in an ordinary superconductor the magnetic permeability vanishes, $\mu=0$, and, consequently, in a dual superconductor $\epsilon=0$. We thus observe that the GribovZwanziger confinement scenario is consistent with the dual Meissner effect proposed as possible confinement 


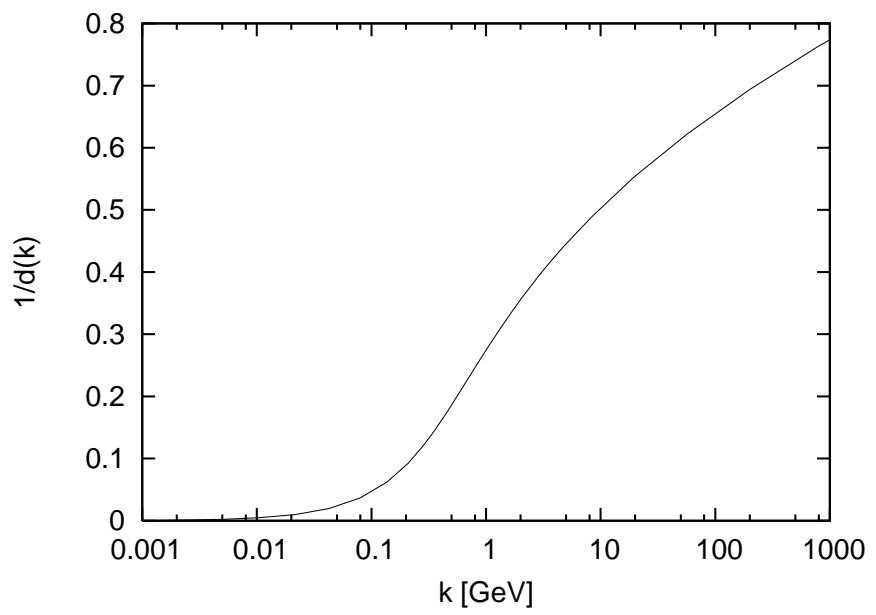

FIG. 1: The dielectric function $\epsilon(\boldsymbol{k})$ (12) of the Yang-Mills vacuum calculated in the variational approach [10] using the solution of the Dyson-Schwinger equations reported in ref. [11].

scenario [4], 15], and supported by lattice calculations [16].

Let us emphasize that the ghost propagator, by definition, depends on the gauge chosen and only the ghost form factor of Coulomb gauge is directly related to the dielectric function by eq. (12).

The ghost form factor $d(\boldsymbol{k})$ has been explicitly calculated in the variational solution of the Yang-Mills Schrödinger equation in Coulomb gauge [10], [11]. Fig. 1 shows the result for the dielectric function (12) obtained from the solutions to the Dyson-Schwinger equations reported in [11]. It has the expected behavior: at zero momentum it vanishes by the horizon condition, while for $k \rightarrow \infty$ it diverges logarithmically due to the anomalous dimension of the ghost propagator. This behavior of $\epsilon(\boldsymbol{k})=d^{-1}(\boldsymbol{k})$ is a manifestation of anti-screening in Yang-Mills theory. (Ordinary Debye screening, which turns the Coulomb potential into a Yukawa potential, produces a dielectric function

$$
\epsilon(\boldsymbol{k})=\frac{m^{2}+\boldsymbol{k}^{2}}{\boldsymbol{k}^{2}},
$$

where $m$ is the inverse screening distance. This dielectric function is divergent at $k \rightarrow 0$ and approaches the QED vacuum value $\epsilon=1$ for $k \rightarrow \infty$ ).

Let us emphasize that the vanishing of the dielectric function in the infrared is a consequence of the horizon condition (13), which is an intrinsic feature of the GribovZwanziger confinement mechanism. This condition has been imposed on the solution to the Dyson-Schwinger equations following from the variational approach [10], [11]. While in $D=3+1$, in principle, solutions to these equations with an infrared finite ghost form factor can be found [17], in $D=2+1$ these equations allow only for solutions satisfying the horizon condition [12]. The ghost form factor in Coulomb gauge has also been calculated on the lattice in both $D=3+1,[18]$, 19] and $D=2+1$ [20]. Unfortunately the lattices used so far in $D=3+$ 1 are not large enough to really penetrate the infrared regime $k<\sqrt{\sigma}$ ( $\sigma$-string tension). In the momentum regime where reliable lattice data are available, there is a reasonable agreement between lattice data [19], 20] and the continuum results [10], 11], [12]. This refers, in particular, to $D=2+1$ dimensions, where larger lattices can be used. The $2+1$ dimensional lattice calculation 20] give strong evidence for an infrared divergent ghost form factor and are in quite satisfactory agreement with the continuum results [12].

In the Zwanziger-Gribov confinement scenario the horizon condition is understood to arise from the field configuration on or near the Gribov horizon, where the Faddeev-Popov kernel develops a zero eigenvalue. One can show analytically 21 that in Landau as well as Coulomb gauge, center vortices and magnetic monopoles lie on the Gribov horizon. The latter configurations are responsible for the dual Meissner effect. Furthermore, when the center vortex configurations are removed from the Yang-Mills lattice ensemble [22], 21] the infrared singular behavior of the ghost from factor is lost. In this sense the Gribov-Zwanziger confinement scenario does not only imply the dual Meissner effect but is also linked to the center vortex condensation picture of confinement.

\section{CONCLUSIONS}

I have shown that the ghost form factor in the Hamilton approach to Yang-Mills theory in Coulomb gauge can be interpreted as the inverse of the dielectric function of the Yang-Mills vacuum. Consequently the horizon condition, a necessary requirement for the Gribov-Zwanziger confinement scenario, implies that in the infrared the Yang-Mills vacuum behaves like a perfect color diaelectric medium, which, in fact, represents a dual superconductor. In this way I have shown that the Gribov-Zwanziger confinement scenario implies the dual Meissner effect.

\section{Acknowledgments}

The author is grateful to W. Schleifenbaum for providing the plot of fig. 1. He also thanks G. Burgio, M. Quandt, W. Schleifenbaum and P. Watson for a critical reading of the manuscript and useful comments. This work was supported in part by DFG under DFGRe856/6-1 and DFG-Re856/6-2.
[1] P. Hasenfratz and J. Kuti, Physics Reports 40 (1978) 75 T.D. Lee, Particle Physics, An Introduction to Field The- ory, (Routledge, New York 1981) 
[2] A. Chodos, R. L. Jaffe, K. Johnson, C. B. Thorn and V. F. Weisskopf, Phys. Rev. D 9 (1974) 3471.

[3] W.A. Bardeen, M.S. Chanowitz, S.D. Drell, M. Weinstein and T.-M. Yan, Phys. Rev. D11 (1975) 1094.

[4] Y. Nambu, Phys. Rev. D10 (1974) 4262

S. Mandelstam, Phys. Lett. B53 (1975) 476

G. Parisi, Phys. Rev. D11 (1975) 970

Z.F. Ezawa and H.C. Tze, Nucl. Phys. B100 (1976) 1

R. Brout, F. Englert and W. Fischler, Phys. Rev. Lett. 36 (1976) 649

F. Englert and P. Windey, Nucl. Phys. B135 (1978) 529

G. 't Hooft, Nucl. Phys. B190 (1981) 455, Phys. Scr. 25 (1982) 133

[5] G. 't Hooft, Nucl. Phys. B138, 1 (1978); G. Mack and V.B. Petkova, Ann. Phys. (N.Y.) 123, 442 (1979); G. Mack, Phys. Rev. Lett. 45, 1378 (1980); G. Mack and V.B. Petkova, Ann. Phys. (N.Y.) 125, 117 (1980) G. Mack, in Recent Developments in Gauge Theories, edited by G. 't Hooft et al. (Plenum, New York, 1980); G. Mack and E. Pietarinen, Nucl. Phys. B205, 141 (1982) Y. Aharonov, A. Casher, and S. Yankielowicz, Nucl. Phys. B146, 256 (1978); J.M. Cornwall, Nucl. Phys. B157, 392 (1979); H.B. Nielsen and P. Olesen, Nucl. .Phys. B160, 380 (1979), J. Ambjorn and P. Olesen, Nucl. Phys. B170, 60 (1980); E.T. Tomboulis, Phys. Rev. D23, 2371 (1981);

[6] For lattice evidence for the center vortex picutre of confinement see: L. DelDebbio, M. Faber, J. Greensite and S. Olejnik, Phys. Rev. D55, 2298 (1997); K. Langfeld, H. Reinhardt, and O. Tennert, Phys. Lett. B419, 317 (1998); M. Engelhardt, K. Langfeld, H. Reinhardt and O. Tennert, Phys. Rev. D61, 054504 (2000); J. Greensite, Prog. Part. Nucl. Phys. 51, 1 (2003)

[7] V. N. Gribov, Nucl. Phys. B 139, 1 (1978).

[8] D. Zwanziger, Nucl. Phys. B 485, 185 (1997) arXiv:hep-th/9603203, Phys. Rev. D70 (2004) 094034.

[9] A. P. Szczepaniak and E. S. Swanson, "Coulomb gauge QCD, confinement, and the constituent representation," Phys. Rev. D 65, 025012 (2002) arXiv:hep-ph/0107078]. A. P. Szczepaniak, "Confinement and gluon propagator in Coulomb gauge QCD," Phys. Rev. D 69, 074031 (2004) arXiv:hep-ph/0306030.

[10] C. Feuchter and H. Reinhardt, "Variational solution of the Yang-Mills Schroedinger equation in Coulomb Phys. Rev. D 70, 105021 (2004) arXiv:hep-th/0408236. C. Feuchter and H. Reinhardt, "Quark and gluon confinement in Coulomb gauge," arXiv:hep-th/0402106
H. Reinhardt and C. Feuchter, "On the Yang-Mills wave functional in Coulomb gauge," Phys. Rev. D 71, 105002 (2005) arXiv:hep-th/0408237.

[11] D. Epple, H. Reinhardt and W. Schleifenbaum, "Confining Solution of the Dyson-Schwinger Equations in Coulomb Gauge," Phys. Rev. D 75 (2007) 045011 arXiv:hep-th/0612241.

[12] C. Feuchter and H. Reinhardt, "The Yang-Mills Vacuum in Coulomb Gauge in $\mathrm{D}=2+1$ Dimensions," arXiv:0711.2452 [hep-th].

[13] H. Reinhardt and D. Epple, Phys. Rev. D76 (2007) 065015, arXiv:0706.0175 [hep-th].

[14] A.L. Fetter and J.D. Walecka, "Quantum theory of many-article systems", Mc. Grow-Hill, New-York (1971)

[15] G. 't Hooft, Nucl. Phys. B190 (1981) 455

[16] A.S. Kronfeld, G. Schierholz and U.-J. Wiese, Nucl. Phys. B198 (1987) 516

T. Suzuki, I. Yotsuyanagi, Phys. Rev. D42 (1990) 4257

S. Hoki et al., Phys. Lett. B272 (1991) 326

G. Bali, Ch. Schlichter, K. Schilling, Prog. Theor. Phys. Suppl. 131 (1998) 645 and ref. therein.

[17] D. Epple, H. Reinhardt, W. Schleifenbaum, and A. P. Szczepaniak, "Subcritical solution of the Yang-Mills Schroedinger equation in the Coulomb gauge," Phys. Rev. D77 (2008) 085007, arXiv:0712.3694 [hep-th].

[18] K. Langfeld and L. Moyaerts, "Propagators in Coulomb gauge from SU(2) lattice gauge theory," Phys. Rev. D 70 (2004) 074507 arXiv:hep-lat/0406024.

[19] M. Quandt, G. Burgio, S. Chimchinda and H. Reinhardt, "Coulomb gauge Green functions and Gribov copies in SU(2) lattice gauge PoS LAT2007, 325 (2007) arXiv:0710.0549 [hep-lat]].

[20] L. Moyaerts, Dissertation, Tübingen, 2004

[21] J. Greensite, S. Olejnik and D. Zwanziger, "Center vortices and the Gribov horizon," JHEP 0505, 070 (2005) arXiv:hep-lat/0407032.

[22] J. Gattnar, K. Langfeld and H. Reinhardt, "Signals of confinement in Green functions of $\mathrm{SU}(2)$ YangMills theory," Phys. Rev. Lett. 93, 061601 (2004) arXiv:hep-lat/0403011.

K. Langfeld, G. Schulze and H. Reinhardt, "Center flux correlation in SU(2) Yang-Mills theory," Phys. Rev. Lett. 95, 221601 (2005) arXiv:hep-lat/0508007.

[23] Note in electrodynamics the coupling constant $e$ is absorbed into the charge units. 